\title{
Microstructure Evolution of Ti Tritides During Aging
}

\author{
Wang Haifeng ${ }^{1}$, Peng Shuming ${ }^{1}$ Shen Huahai ${ }^{1}$ and Zhou Xiaosong $^{1}$ \\ ${ }^{1}$ Institute of Nuclear Physics and Chemistry, China Academy of Engineering Physics, Mianyang, China
}

Metal tritides are used for stable, high-density storage of tritium. The behavior of helium in metal tritides is of interst because ${ }^{3} \mathrm{He}$ is produced by the radioactive decay of tritium. Helium accumulates in metal tends to precipitate into bubbles. During the first few years of storage, He is released at less than about $1 \%$ of the generation rate from many tritides. When the helium concentration reaches a critical value, helium is released at rates equal to and sometimes exceeding the generation rate. The size, density and distribution of helium bubbles is the main parameters controlling the helium bubble evolution during the aging of metal tritides. The object of this paper is to study the microstructure of helium bubbles in the Ti tritides by TEM.

Titanium films were deposited by resistively-heated evaporation to a thickness of several micrometers on the molybdenum substrates. the sample then were loaded with tritium gas to produce tritide films with the initial composition of $\mathrm{TiT}_{(1.7 \sim 1.9)}$, the sample were stored in a vacuum vessel at room temperature, the released Helium during the storage were measured by a Quadrupole Mass Spectrometer (QMS). The cross-sectional TEM samples were prepared by focused-ion beam instrument (FIB). TEM analysis was performed using a FEI F30 $300 \mathrm{keV}$ TEM.

TEM observations have been performed on Ti tritides at He:Ti ratios of 0.03-0.35. The sample with He:Ti ratios of 0.35 is at the helium accelerated release stage. Helium bubbles are shown as no less than $1 \mathrm{~nm}$ with uniform distribution after several months aging. The size of helium bubbles in all the samples is between $0.5-0.9 \mathrm{~nm}$, the mean size in all the sample is around $0.7 \mathrm{~nm}$. The size and spherical shape of bubbles remain unchanged on the whole storage period, but the density of helium bubbles is increase linearly with aging time. It can conclude that the process of nucleation continues throughout the whole storage of Ti tritide. The biggest helium bubbles with size over 0.8 are common seen in young sample, hardly found in old sample. The grow-up helium bubbles seems have not competitive advantage on acquiring free helium atoms. Das et al have calculated the effect of helium clusters on the perfect and Pd vacancy-containing $\mathrm{PdT}_{0.47}$ and $\mathrm{PdT}_{0.72}$ lattices. The formation energy per atom is decreasing with increasing cluster size [1]. This means the migration of helium atoms prefer the new generated helium bubbles to the grow-up helium bubbles, and only the single isolated helium bubbles mostly lie in young sample can grow up into a big one. No strain contrast is visible extend outside the perimeter of the bubble in this study, since Johnson suggested that an overpressured bubble may sets up a strain field in the surrounding matrix [2]. Perhaps the strain contrast is only lie in single isolated overpressured bubble, and in our study helium bubbles are affected by the backpressure from the presence of neighboring bubbles.

The density of the bubbles is in the range of $1-6 \times 10^{24}$ bubbles $/ \mathrm{m}^{3}$. Since the released ${ }^{3} \mathrm{He}$ are less than $0.1 \%$ total helium production, nearly all the ${ }^{3} \mathrm{He}$ produced are in the films. The mean size of helium bubbles is around $0.7 \mathrm{~nm}$, it can deduce from the the equation of state (EOS) that the helium bubbles may in too high pressure (over $10 \mathrm{GPa}$ ), but no significant strain contrast is observed. This result has also been found in other tritides $(\mathrm{Lu}, \mathrm{Nb}, \mathrm{Pd}$ and $\mathrm{Ta}$ ). Thiebaut deduce that only a fraction of the helium is 
localized within the bubbles [3]. But most researchers think all the helium are in helium bubbles. To figure it out, we comparing the images of sample with and without tilt $4^{\circ}$, The contrast of the helium bubbles have great changes and some helium bubbles disappear, some new helium bubbles appear. When observing the sample through the other side, all the helium bubbles are renewed, only several helium bubbles could be figured out, but their intensities faded very much. Thiebaut had simulate the bubble contrast in Pd tritides showed that the contrast is affected by the position of the bubble in the sample thickness, It increase when the bubble is close to the lower surface of the specimen [3]. This means only helium bubbles close to the one side of surface are observed by TEM, and there are many more helium bubbles have not been observed in the current TEM analysis technique. Considering the loss of helium from both surface of samples, the density of helium bubbles obtain by TEM should be increased by several times to one order of magnitude.

The behavior of Helium bubbles in Ti tritides have been studied by TEM, the results show that Helium bubbles with less than $1 \mathrm{~nm}$ in diameter are uniform distribution for the whole storage period. The size and spherical shape of bubbles remain unchanged on the whole storage period, but the density of helium bubbles is increase linearly with aging time. The density of the bubbles is in the range of $1-6 \times 10^{24}$ bubbles $/ \mathrm{m}^{3}$. By comparing the images acquired in tilting and turning over samples, the density of the helium bubbles may be higher up to an order of magnitude.

\section{References:}

[1] N K Das and N H de Leeuw, J. Phy.: Condens. Matter 27 (2015), p. 475002.

[2] P. B.Johnson and D. J. Mazey, J. Nucl. Mater. 111/112 (1982), p. 681.

[3] Thiébaut_et al, J. Nucl. Mater. 217 (2000), p. 277.
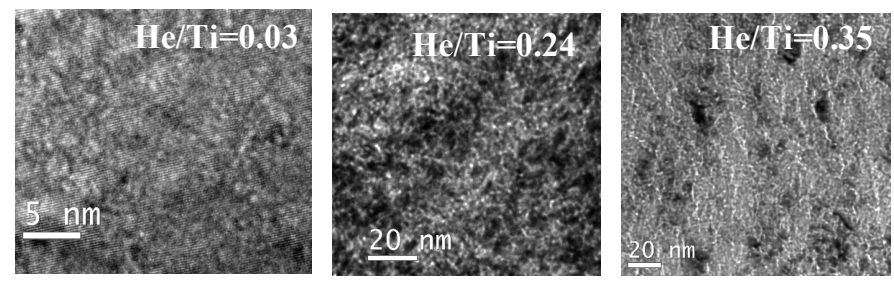

Figure 1. Bright-field images from samples at He:Ti ratios of (a) 0.03, (b) 0.24, (c) 0.35.
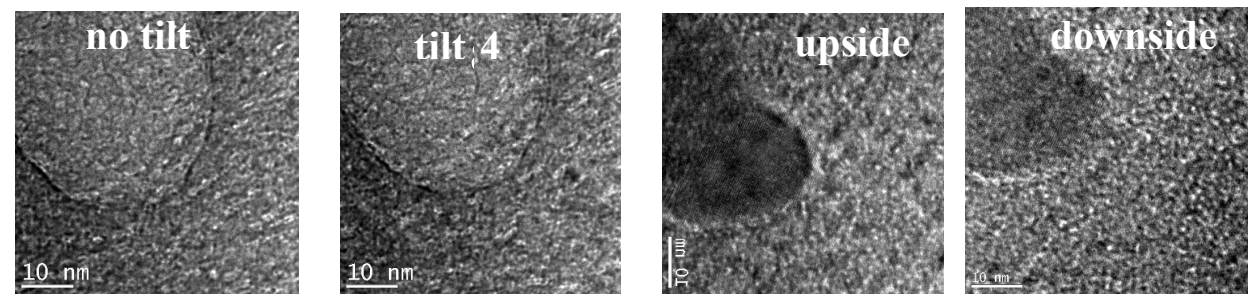

Figure 2. Images by tilting the sample with and without $4^{\circ}$.

Figure 3. Images viewed from upside and downside of the sample. 